
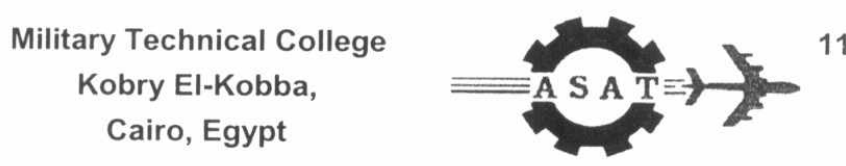

11-th International Conference on Aerospace Sciences \& Aviation Technology

\title{
PERFORMANCE OF OIL-INJECTED TWIN-ROTORS SCREW COMPRESSOR
}

\author{
KASSAB" S. Z., ADAM"'I. G. AND ELGOHARY"** H. A.
}

\begin{abstract}
In order to improve the performance of screw compressor, it is important to know, understand, and analysis the different geometrical as well as operating parameters on the compressor performance. Consequently, the present study is concerned with obtaining the characteristics of twin-screw compressor by using a simulation code named SCORPATH. Explanation of the code is presented and discussed.

A parametric study was performed to determine the effect of the geometrical and the operating parameters on the performance of screw compressor. The study revealed that the geometrical parameters: clearance gap, number of lobes in the rotors, rotor relative length, and screw wrap angle, have significant effect on the performance of the screw compressor. In addition, built in volume ratio, tip speed, suction and discharge pressures, are the operating parameters which have a great effect on the performance of the twin-screw compressor.
\end{abstract}

\section{Keywords:}

Compressor, screw machine, oil-injected.

*Professor, Mechanical Engineering Department, Alexandria University, Egypt.

**Lecturer, Mechanical Engineering Department, Alexandria University, Egypt.

${ }^{* \star *}$ Graduate Student, Mechanical Engineering Dept., Alexandria University, Egypt. 


\section{NOMENCLATURE}

A Cross section area through which the working fluid passes.

D Rotor diameter

h Specific enthalpy.

$L \quad$ Length of the screw rotor of the compressor.

$\dot{m}$ Mass flow rate of the working fluid through the rotor.

$N \quad$ Rotational speed (rpm).

$P$ Power absorbed or resulting from the screw rotors of the compressor.

$p \quad$ Fluid pressure in the control volume.

$\dot{Q} \quad$ Heat transfer between the fluid and the compressor surrounding.

$R \quad$ Fluid Constant

$T$ Temperature.

$t$ Time.

$U \quad$ Internal energy of the working fluid.

$\checkmark \quad$ Instantaneous volume of the compressor working chamber.

$W \quad$ Work transferred to or from the rotors of the compressor.

$z_{1} \quad$ Number of working volumes of the main screw lobes.

\section{Greek Symbols}

$\begin{array}{ll}V & \text { Adiabatic exponent } \\ \eta_{v} & \text { Volumetric efficiency } \\ \eta_{T} & \text { Total efficiency. } \\ \rho & \text { Working fluid density. } \\ \Pi & \text { Pressure ratio }\end{array}$

\section{Subscripts}

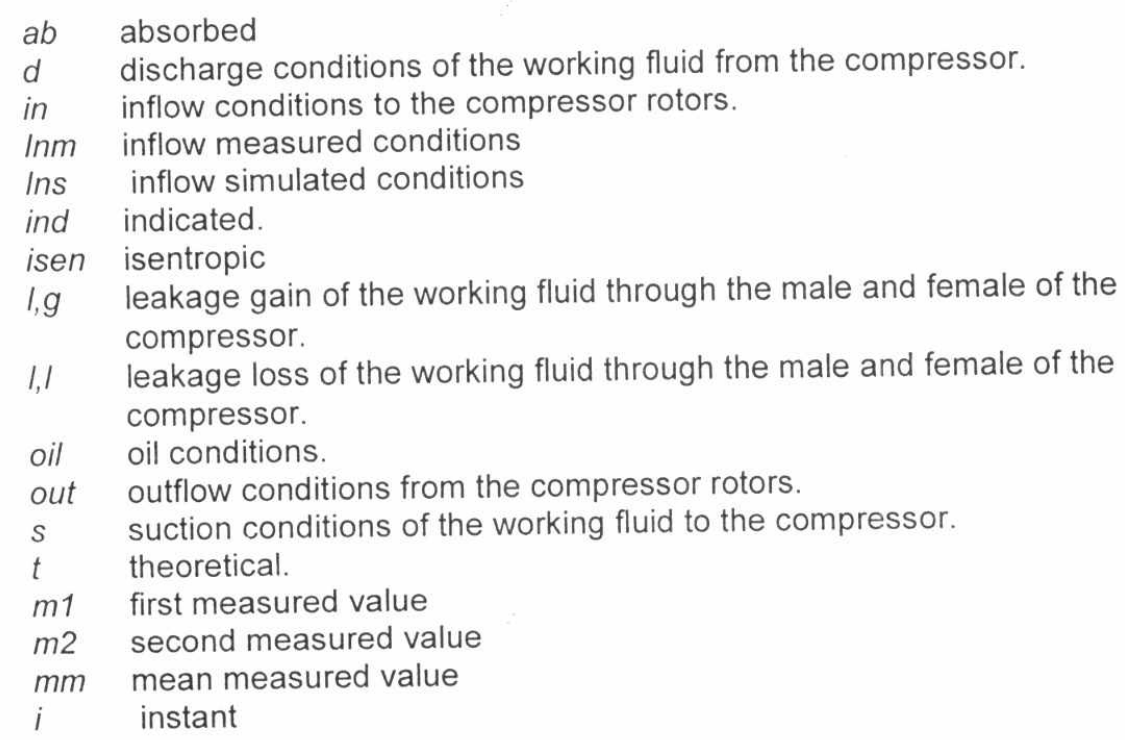




\section{INTRODUCTION}

Screw compressor, Fig. 1, is one of the rotary positive displacement machines of simple design able to work with high speed operation over a wide range of operating pressures and flow rates with high efficiencies. It is compact and reliable. The screw compressor, Fig. 2, has the same performance characteristics as any positive displacement compressor. It traps a mass of gas in a sealed chamber. The gas is then compressed as the chamber volume is reduced by the conjugate helical surface with complex transverse tooth. The entire flow volume is discharged after compression with no clearance pockets.

The characteristics of the twin helical screw compressor make it more attractive to users than either the large reciprocating piston compressor or the small centrifugal compressor. Due to the development of advanced machine tools, the mass production of a high quality rotor pair, the essential feature of an efficient and reliable and maintenance-free twin helical screw compressor had made possible. Its low wear rate, due to non-contact in one variant and high lubrication in the other, meaning that its operating characteristics in service remain the same over long periods.

Screw compressors are used for a variety of applications. They are used for the compression of a vast range of gases and vapors including refrigerant. Also, they are used in the industrial process, the oil and gas industrial and for supercharging the internal combustion engines of road vehicles. For optimum performance, a specific design and operating mode is need for each application. In the case of refrigeration plant, they are expected to operate efficiently over a wide range of loads for long periods.

Screw compressor is produced in two variants, oil-free and lubricated. One of the main features of the early screw compressor was the oil free. The oil-free compressor has seals, which prevent oil mixing with the compressed gas. One rotor drives the other through timing gears, which synchronize their rotation without physical contact lobe to lobe to casing. In some applications water or a liquid solvent is injected into the compression cavity to limit the discharge temperature, to prevent premature polymerization or simply to wash away contaminating particulate entrained in the gas stream. In the lubricated compressor type lubricating oil enters the bores from the bearings and in some compressors by direct injection into the compression cavity. The oil improves sealing and cooling and permits the mechanical simple arrangement of one rotor driving the other through lubricated lobe-to-lobe contact. The oil is required to be compatible with the gas being compressed. As a consequence, synthetic lubricants are often used in preference to mineral oils.

The original idea for a screw blower was embodied in a German Patent granted to Heinrich Krigar of Hannover as early as 1878. The first practical screw compressor was not developed until the early $1930 \mathrm{~s}$, the Swedish engineer, Alfred Lysholm, later patented it. Lysholm's compressor, which was three lobes on the male rotor, three flutes on the female rotor $(3+3$ profile combination), was produced in 1937. By 1937 a 4-6 profile combination compressor had been developed. In 1938 
such a compressor was manufactured, in collaboration with Lysholm, by James Howden and company of Glasgow, Scotland. The first developed work was described in the Proceeding of the Institution of Mechanical Engineers, 1943. From this work was derived the most widely applied form of screw compressor for gas compression used today. In more recent times the Ljungstrum Company was renamed Svenska Rotor Maskiner AB (SRM). Most screw compressors for process gas compression today are manufactured under license from this company, [1].

Despite the rapid growth in screw compressor usage, public knowledge of the scientific basis of their design is still limited. Chung-Bian and Shinn-Liang[2] developed a mathematical model of the milling cutter of worm surface. While Fleming et al. $[3,4]$ developed a computer program that generates profiles with the ability to change any working parameter. Further, Da Zhun Xiao and Ke Bing Li[5] developed a computer aided design software package for conjugate helical surfaces applied to the twin-screw compressor. Stosic and Hanjalic[6,7] presented a method for the design of twin-screw compressor and expanders which are based on a differential algorithm for defining the rotor profiles. Moreover, They produced SCORPATH, it is a universal computer program that generates any profile. Stosic[8] discussed the manufacturing way of the screw and how close they are to helical gear. The envelope gearing method is used to derive a meshing condition for crossed helical gear. A reverse transformation enables the rotor profile thereby manufactured to be calculated.

Zimmen and Sweetser[9] considered the centrifugal economizer and its match with screw compressors showing how much gain can be earned by this combination. Also, Pillis[10] showed how using screw compressor increases the efficiency and durability of a refrigeration cycle.

Fleming et al.[4] presented an analysis of the working process. They generated a simulation program considering only the refrigeration screw compressor. They studied the thermodynamic effects of internal leakage of gas through all paths, oil injected and drained and refrigerant injection.

Performance factors for screw compressors are both volumetric and total efficiencies. Volumetric efficiency reflects the leakage through the compressor element and the accuracy of the machining of the screw rotors with their housing. Total efficiency represents a faithful judgment on the performance of the machine either it needs highly input power relative to its output or its effectiveness is high. Another two important performance factors are the mass flow and the input power. The mass flow provides the designer with a real scope on the applications for which the compressor can be used and the configuration of the compressor for the need of a certain mass flow. Meanwhile, the input power gives the designer an overview over his/her design either it is not effective enough to be produced or he/she is working in the correct way for a better design.

Factors that influence the performance of a twin screw compressor may be grouped into two categories as follows:

- The geometrical parameters, such as clearance gap, number of teeth in the main and gate rotors and rotor relative length. 
- The operating parameters, such as built in volume ratio, tip speed of the rotors, suction and discharge pressures.

The present investigation is directed to study the performance of oil-injected twinrotors screw compressor, working at full load, using a computational code. A mathematical analysis of the working process is presented. The effect of various geometrical and operating relevant parameters on the performance of the screw compressor are presented and discussed.

\section{MATHEMATICAL FORMULATION}

\section{Profile generation}

The present rotor configuration $4 / 6$ (male/female teeth ratio), is one of a family of rack generated involute ' $N$ ' profiles designed for the efficient compression of air, common refrigerants and process gases. The basic idea used to generate the rotor profiles, is to produce primary profile curve of one rotor by dividing the profile into small segments of circular arcs and straight lines, then generate a corresponding secondary profile curve of the other rotor by using an appropriate conjugate criterion. More details can be found in Stosic and Hanjalic[6].

\section{Mathematical formulation}

The energy conservative equation and the continuity equation, in conjunction with auxiliary equations defining the leakage, are utilized to study the flow process in a screw compressor. The conservation of internal energy is given by

$$
\frac{d U}{d t}=(\dot{m} h)_{\text {in }}-(\dot{m} h)_{\text {out }}+\dot{Q}-p \frac{d V}{d t}
$$

where $U$ is the internal energy, $h$ is the specific enthalpy, $\dot{m}$ is the mass flow rate, $p$ is the fluid pressure, $\dot{Q}$ is the heat transfer between the fluid and the surrounding, and $V$ is the instantaneous volume of the compressor working chamber. The subscripts "in" and "out" are stand for the inflow and outflow respectively. The inflow and outflow fluid total enthalpy is expressed as

$$
(\dot{m} h)_{i n}=(\dot{m} h)_{s}+(\dot{m} h)_{l, g}+(\dot{m} h)_{o i l, i n} \text { and }(\dot{m} h)_{o u t}=(\dot{m} h)_{d}+(\dot{m} h)_{l, l}
$$

where the subscripts "I,g" and "I,I" denote the leakage gain and leakage loss, " $s$ " and " $d$ " indicate the suction and discharge conditions and "oil,in" represents the injection oil. 
The mass continuity equation is

$$
\frac{d m}{d t}=\dot{m}_{\text {in }}-\dot{m}_{\text {out }}
$$

The inflow and outflow masses is given by

$$
\dot{m}_{\text {in }}=\dot{m}_{s}+\dot{m}_{l, g}+\dot{m}_{\text {oil }} \quad \text { and } \quad \dot{m}_{\text {out }}=\dot{m}_{d}+\dot{m}_{l, l}
$$

The above conservation equations, together with constitutive equations describing the leakage phenomena and the fluid state, are solved using a computer package "Screw COmpressors Rotor Profiling And THermodynamics (SCORPATH)" developed by Stosic and Hanjalic[7]. The "SCORPATH" program use an iterative numerical procedure to obtain the mass, pressure and temperature as a function of the rotation angle. In order to estimate the overall screw compressor performance, the results are integrated over the cycle.

The numerical analysis of the physical process provides detailed results of important properties such as; the mass flow rate, $\dot{m}(\mathrm{~kg} / \mathrm{s})$, the volumetric efficiency, $\eta_{v}$, input power $(\mathrm{kW})$ and total efficiency, $\eta_{T}$. The theoretical mass flow is calculated from:

$$
\dot{m}_{t}=\frac{\left(A_{1}+A_{2}\right) L \rho z_{1} N}{60}
$$

where $A_{1}$ and $A_{2}$ are the cross section areas of the lobes in the front plane of the main screw and the gate screw respectively, $L$ is the length of the screw, $z_{1}$ is the number of working volumes of the main screw lobes, contributing towards the process. Hence, the volumetric efficiency is obtained as:

$$
\eta_{v}=\frac{\dot{m}}{\dot{m}_{t}}
$$

The leakage effect is included through the differential treatment of the governing equations.The indicated work transferred to the screw rotors during the suction, expansion and discharge processes equals to the area of the indicated $p-V$ diagram. Hence, the indicated work and power are calculated from:

$$
\begin{gathered}
W_{\text {ind }}=\int V d p \\
P_{\text {ind }}=\frac{W_{\text {ind }} z_{1} N}{60}
\end{gathered}
$$


The isentropic and absorbed power are then obtained from:

$$
\begin{gathered}
P_{i s e n}=\frac{\gamma}{\gamma-1} \dot{m} R T_{i n}\left(\left(\frac{p_{s}}{p_{d}}\right)^{\frac{\gamma-1}{\gamma}}-1\right) \\
P_{a b}=P_{\text {ind }}+\text { mechanical losses }
\end{gathered}
$$

The total efficiency is readily obtained as:

$$
\eta_{T}=\frac{P_{i s e n}}{P_{a b}}
$$

The built in volume ratio $\left(V_{i}\right)$, which is the ratio of the trapped volume at the instant the edge of the inlet ports closes the space to the volume of the space at the instant the edge of outlet port opens the space, is related to the pressure ratio $(\pi)$ by the following equation:

$$
\pi=V_{i}^{K}
$$

where $K$ is the isentropic exponent

\section{VALIDATION}

Validation of the used code is done by comparing its output results with measured results to know how practical the code is and to locate the points of development for better code performance. This approach reduces the time and cost required for a good design. This is especially true of an industrial compressor, which must be connected to a process plant, which by its nature has a long setting time .

It was remarked that the used code in the present study has limited reference with experimental results, there is no comparison between the $4 / 6$ profile and the experimental measured data, the only comparison, available in the literature [6\&7], is for the $5 / 6$ profile, so that user does not have sufficient insurance of his work corresponding to the practical model. It was necessary then to make a further comparison with experimental data available in the literature.

Comparing computer work with experimental results, declares a difference that either is limited to be acceptable which results in availability of the code or large to be refused and get in some developments and correction factors to eliminate the gap between experimental results and those of the code. Also any working process simulation program can not on its own, be used to replace the physical prototype 
tests entirely since important coefficients in the mathematical model are determined by some test results. Actually, developments are used when there is large difference between the two sets of results (code and experimental). Consequently it is needed to make some modifications in the programming procedure for better results. For correction factors, they are used in case of medium difference so that the programming procedure is correct but not accurate due to several effects which is natural. Sometimes some correction factors can not be predicted or evaluated mathematically but they need to evaluate by certain test that make most of the modifications very difficult .

Validation of the applied numerical code is carried out through a certain sequence. Practically speaking as designers generate profile to realize the desired working conditions, it is logic first to validate the profile generation of the used package, then pass to the validation of the thermodynamic analysis of the working process

A comparison was carried out by Elgohary [11], between the generated profile by the used code and the measured profile supplied by Howden Compressor Company data book presented in [1]. A good agreement between the computed and measured profiles was obtained. Hence, based on this comparison, it can be concluded that the present simulation code has an accurate profile generation upon which designers can depend to execute a high precision work saving their time.

In order to validate the present thermodynamic results, a comparison is made with the Howden Compressor Company data[1]. The configurations of Howden Compressor machine are:

- Working fluid is refrigerant R22.

- Rotor lobe profile- SRM D standard.

- The superfeed is not on duty.

- No liquid refrigerant injected.

- Oil drained and injected.

- Compressor is running on full load.

- Oil injection is applied

The working conditions are:

- Male and female rotor diameters equal $204 \mathrm{~mm}$.

- Wrap rotor angle: 300 .

- Length/diameter ratio: 1.65.

A comparison between the simulated and two sets of the measured volumetric efficiency are presented in Table 1. It is clearly presented how close are the simulated volumetric efficiencies to the mean measured values $\left(\eta_{\mathrm{vmm}}\right)$. The maximum difference reaches $2.1 \%$ at pressure ratio equals 5.27 .

The most important comparison parameter is the absorbed power. Table 2. presents a comparison between the simulated and measured absorbed power. The 
difference between the values is small and can reach a maximum of about $3.6 \%$. The comparison demonstrates the accuracy of the used code.

Table 1. Comparison between both simulated and measured volumetric efficiency

\begin{tabular}{|c|c|c|c|c|c|c|}
\hline Case & $\begin{array}{c}\text { Pressure } \\
\text { ratio }\end{array}$ & $\begin{array}{c}\eta_{\mathrm{vm} 1} \\
\%\end{array}$ & $\begin{array}{c}\eta_{\mathrm{vm} 2} \\
\%\end{array}$ & $\begin{array}{c}\eta_{\mathrm{vmm}} \\
\%\end{array}$ & $\begin{array}{c}\eta_{\mathrm{v}} \\
\%\end{array}$ & $\begin{array}{c}\text { Difference } \\
\%\end{array}$ \\
\hline 1 & 3.0 & 92.8 & 91.2 & 91.9 & 92.4 & 0.5 \\
\hline 2 & 4.25 & 91.2 & 91.2 & 91.2 & 92.9 & 1.9 \\
\hline 3 & 5.27 & 92.3 & 89.5 & 90.9 & 92.8 & 2.1 \\
\hline 4 & 6.41 & 92.3 & 91.2 & 91.8 & 92.7 & 1.1 \\
\hline
\end{tabular}

Table 2. Comparison between both simulated and measured input power

\begin{tabular}{|c|c|c|c|c|}
\hline Case & $\begin{array}{c}\text { Pressure } \\
\text { ratio }\end{array}$ & $\mathrm{P}_{\text {inm }}$ & $\mathrm{P}_{\text {ins }}$ & $\begin{array}{c}\text { Difference } \\
\%\end{array}$ \\
\hline 1 & 3.0 & 189.1 & 195.8 & 3.6 \\
\hline 2 & 4.25 & 165.9 & 171.4 & 3.3 \\
\hline 3 & 5.27 & 157.4 & 159.9 & 1.7 \\
\hline 4 & 6.41 & 151.3 & 147.4 & -2.6 \\
\hline
\end{tabular}

The results presented in Tables 1 and 2 show that the applied code provides good results with respect to the experimental results. The deviation between the two results is due to several factors. The relation between the built in volume ratio and the pressure ratio can be one of those factors because the inaccurate value of the isentropic exponent may lead to improper values. Also the relation of the built in volume ratio and the internal pressure ratio could not be applicable with convinced accuracy for high pressure ratios as the pressure difference between the discharge pressure and the pressure in the control volume becomes large, the gas flow can not be considered as incompressible because the Mach number could be quiet large .

Another cause of the results deviation is the sensitivity of the measuring tools and devices, also the mechanism of taking the measures has a great effect on the value registered as the way of using and placing the thermocouple for reading the temperatures. These phenomena explain why manufacturer presents data in several values for the same conditions. 
The gap between the simulated and the measured volumetric efficiency is due to the absence of some factors. Area coefficient represents one of them, because it provides an area change caused by the difference between the calculated and the real areas. Also the value of this coefficient is the subject of discussion, each manufacturer decides it's value upon his fabrication facilities. In addition, some other factors are the tendency of the oil in the compressor to seal the leakage gaps, the changes caused to nominal clearances by the cavity pressure under load and by the temperature distribution of the rotors and housing under load. Consequently the area coefficient is categorized to depend mainly on circumstances and do not have a constant value. On the other hand, the accuracy of timing the control of the suction and discharge port is very important and need to some correction factors evaluated experimentally which are treated as manufacturing secrets. The optimum closing angle of the suction port depends on the balance between the gas sucked and the gas leaking into the cavity during the last stage of the suction process and the optimum closing angle where the flow speed equals zero at the end of the suction process which will lead by its turn to a loss in the volumetric efficiency.

The radial and axial clearances gaps values do vary according to the load on compressor because of material enlargement due to heat stress and the deflection of the rotor causing the gaps to increase or decrease. These two parameters are taken constant in the simulation procedure, which get by its turn to a different in volumetric efficiency.

The results presented in Table 1 demonstrate that the contribution of the above mentioned factors is small. This is judged by the small maximum difference between the measured and predicted values for the volumetric efficiency of about $2.1 \%$. These results give confidence to the use of the applied code to predict the compressor performance.

A great attention should be paid while remarking the difference between both the calculated and measured absorbed power. The deviation in the results is due to the influence of a list of factors such as oil injection and the type of the oil, built in volume ratio, speed coefficient in the computation of the mass flow, the pressure difference, the friction power losses calculation, the relation between the refrigerant and oil and the heat transfer either between oil and refrigerant and between compressor and the surrounding.

The way of calculating the friction power loss is another direct effect in the deviation subject of the study. Through the analysis friction losses is kept constant at $10 \%$ as an average rate[1]. Practically speaking this loss result from the end casings, from shaft seal, radial and thrust bearings and the balance pistons. They also result from the main casing such as the friction losses due to the presence of oil in small gaps and due to the viscosity of the compressed gas. In a refrigerant screw compressor the friction losses from end casings have an indirect influence on the working process in the main casing. The lubrication oil is heated due to the friction losses. The bearing power losses and the shaft seal losses are calculated using bearing and shaft seal loss data or charts supplied by the manufacturers. 
Additional deviation factor is the solubility of refrigerant and lubricating oil. When the refrigerant dissolved in the oil reduces the viscosity of the oil and worsens the sealing between the gaps and it is a source of additional volumetric and energy losses in the compressor.

Also the heat exchanged with the gas in the control volume could be an important factor having a great influence on the behavior of screw compressor. Usually the discharge temperature is measured in the discharge line just after the discharge chamber, which is not real discharge temperature due to the presence of an oil film on the sensing element of the thermometer. Also the material of the compressor housing and the accuracy of its manufacturing affects directly the heat transfer and by its turn affects the energy equation leading to a difference between the actual case and the simulated one.

Based on the previous results, it is quiet safe to use the present code in the design and optimization of an universal screw compressor because the deviation of the performance of this code from the experimental and test case is located, analyzed and limited.

\section{RESULTS AND DISCUSSIONS}

\section{Effect of geometrical parameters}

Profile factors have been kept constant for each study except the studied parameter. They have the following values:

Center distance $=0.3214 \mathrm{~m}$; Radii of main rotor tooth $R_{1}=0.111 \mathrm{~m}, R_{2}=0.00344 \mathrm{~m}$; Gate rotor radii $R_{3}=0.00258 \mathrm{~m}, \mathrm{R}_{4}=0.01431 \mathrm{~m}$; Radial and axial clearance $\mathrm{GAP}_{1}=$ $0.000295 \mathrm{~m}, \mathrm{GAP}_{2}=0.000295 \mathrm{~m}$; Rotor relative length $=1.65$; Eccentricity of the rotor tooth $=0.00 \mathrm{~m}$; Number of teeth in the main and gate rotor $=4 / 6$; Profile teeth tip angle $=0.0041$ rad; Contact belt pressure angle $=0.3,0.6,0.4$ rad; Angle of the computation start $=0.00 \mathrm{rad}$; Screw wrap angle $=5.23 \mathrm{rad}$; Angles of the compression start and compression end $=0.00$ rad, 4.00 rad respectively; Built in volume ratio $=2.2$.

Figure 3-a presents the variation of mass flow rate with the clearance gap (the distance between the rotor and the compressor housing) for various profiles configurations. The results show that as the gap increases the mass flow rate linearly decreases for all profile configurations. This behavior is due to the increase of the leakage through the wide gap, which leads to a decrease in the mass flow through the compressor. With reference to mass calculations, one can say that, for the same clearance gap, by increasing the number of lobes the mass will increase also. This is clearly shown in Fig. 3-a. Based on the definition of the volumetric efficiency as the leakage increases the volumetric efficiency decreases. This is clearly reflected in Fig. 3-b. As the number of lobes increases the theoretical and actual mass flow rate increase. Meanwhile, the increase of the cross sectional area 
of the lobes leads to the increase of the theoretical mass flow. Consequently, the volumetric efficiency will be decreased by the increase of the number of lobes. Moreover, Fig. 3-b shows that the rate of drop in the volumetric efficiency increases with the increase of the number of lobes. This is due to the increased leakage that happens through the clearance gap for higher profile configurations.

For any decrease in the mass flow rate, indicated power also decreases. As the input power is the result of both mechanical power loss and the indicated power, so the input power will also decreases with the decrease of the mass which can be easily remarked from Fig. 3-c. In addition, for higher number of lobes, the input power increases as the mass flow rate increases. Figure 3-d shows that, for certain profile configuration, as the clearance gap increases the total efficiency decreases. Further, for certain clearance gap, as the input power increases for higher number of lobes, the total efficiency decreases for higher number of lobes than for smaller ones. The drop in the total efficiency between $3 / 5$ and $5 / 7$ profiles increase by the increase of the clearance gap. This is caused due to the decrease in the mass flow, which reaches its maximum value by the larger clearance gap.

The number of teeth in the main is the number of lobes on the main rotor. It is coupled with the number of gates on the female rotor. In the present study three categories were considered. These are $3 / 5,4 / 6$ and $5 / 7$. Using the computation code, the results presented in Table 3 demonstrating the effect of varying the number of teeth in the main rotor on the input power, mass flow, total and volumetric efficiencies. From this table it is found that increasing the number of lobes to gates results in an increase in the mass flow rate. Further, increasing the number of lobes and gates means that the compression volume is also increased. Consequently, the isentropic power increases resulting in a decrease in total efficiency. In addition, the volumetric efficiency also decreases. All these effects are clearly displayed in Table 3.

Table 3. Effect of the number of main-rotor teeth on compressor performance.

\begin{tabular}{|c|c|c|c|c|c|}
\hline Case & Main/Lobe & $\begin{array}{c}\text { Mass Flow } \\
\mathrm{kg} / \mathrm{s}\end{array}$ & $\begin{array}{c}\text { Input Power } \\
\mathrm{kW}\end{array}$ & $\begin{array}{c}\text { Volumetric Eff, } \\
\%\end{array}$ & $\begin{array}{c}\text { Total Eff. } \\
\%\end{array}$ \\
\hline 1 & $3 / 5$ & 3.5 & 45.45 & 94.7 & 56.9 \\
\hline 2 & $4 / 6$ & 4.6 & 46.69 & 92.6 & 55.7 \\
\hline 3 & $5 / 7$ & 5.6 & 48.30 & 91.5 & 53.9 \\
\hline
\end{tabular}

The rotor relative length, $L / D$, is the ratio between the length of the rotor, $L$, and its diameter, D. As the compressor rotor diameter is constant through the present study the change in the relative length leads to a change in the rotor length. Figure 4-a shows the variation of mass flow rate with the relative length. Increasing the relative length the mass flow rate is linearly increased due to the absorption of more amount of mass. For certain L/D ratio, Fig. 4-a shows also that as the number of lobe to gate increases the mass flow rate increases. Meanwhile, Fig. 4-b shows that, for certain configuration, as the rotor relative length increases the volumetric efficiency increases. Moreover, the difference between the volumetric efficiency for $3 / 5$ profile 
and $5 / 7$ for small L/D ratio justified due to large leakage in case of $5 / 7$ profile relative to the suction volume and the small leakage as the number of lobes reduced.

As the mass flow rate increases, this leads to an increase in the isentropic power and the total efficiency will vary as a result taking into consideration the variation of the input power. Input power will also be increased by its turn due to the increase of the load on the compressor rotors. Figures 4-c and 4-d demonstrate these relations. The difference between the total efficiency for $3 / 5$ profile and $4 / 6$ for small ratio is large and this difference begins to decrease until it reaches its minimum for large L/D ratio. This is due to the small input power of $3 / 5$ configuration, which results in higher total efficiency. Moreover, the leakage is small for small L/D ratio beside the small configuration of the compressor.

Figure 5-a shows the variation of the mass flow rate with respect to screw wrap angle. The screw wrap angle is the angle by which the element of the compressor is screwed when manufactured. Increasing the screw wrap angle leads to decrease in the mass flow rate. This is due to the change in the compression chamber. Coupled with this decrease, a similar decrease takes place in the volumetric efficiency as shown in Fig.5-b.

For a decrease in the shape of the compression chamber, the mass flow rate will decrease leading to a decrease in the input power due to a reduction volume. Figure $5-c$ reflects this relation. On the other hand, the total efficiency presented in Fig. 5- $d$ increases with the increase of the screw wrap angle reaching a maximum value before it starts to decrease in the case of $4 / 6$ and $5 / 7$ profiles.

\section{Effect of operating parameters}

In the present study thermodynamic factors kept constant for each study except the studied parameter. They have the following values:

Tip speed $=64.18 \mathrm{~m} / \mathrm{s}$; Suction pressure = 1bar; Discharge pressure $=2$ bar; Suction temperature $=268 \mathrm{k}$; Prespecified discharge temperature $=340 \mathrm{k}$; Evaporating temperature $=268 \mathrm{k}$; Condensing temperature $=298 \mathrm{k}$; Compressor housing mean temperature $=0.00$; Vapor quality on inlet $=1.00$; Fluid choice switch = 8; Gas constant 1.1; Adiabatic exponent = depend on fluid; Real gas factor $=0.00$; Oil to gas mass ratio $=0.02$; Specific heat of oil $=2050 \mathrm{~J} / \mathrm{kg} . \mathrm{k}$; Oil density $=950$ $\mathrm{kg} / \mathrm{m}^{3}$; Oil inflow temperature $=377 \mathrm{k}$, Oil injection angle $=1.9 \mathrm{rad}$; Mean sauter oil droplet diameter $=0.00001 \mathrm{~m}$.

Figure 6-a presents the relation between the mass flow rate and built in volume ratio. As it appears mass flow rate is almost constant over the whole range. In addition, this figure shows that as the profile configuration increases the mass flow rate increases. This is due to the greater size of the compressor. Meanwhile, Fig. 6-b shows that as the lobe number increases there is a decreasing effect on the volumetric efficiency with the increase of the built in volume ratio. Any increase in the built in volume ratio is coupled with an increase in the pressure ratio leading to a slight increase in isentropic power which is not as large as the input power increase 
that is shown in Fig.6-c. Consequently, this results in a sensible decrease in the total efficiency with the increase of the built in volume ratio. Figure 6-d demonstrates this relationship.

The tip speed of the rotor is the speed with which the main rotor runs. The effects of varying this parameter on the compressor performance are presented in Fig. 7. Figure 7-a shows that with the increase in tip speed the mass flow rate of the compressor linearly increases. This is physically correct because as the speed increases the number of formation of a compression chamber increases. Meanwhile, Fig. 7-b shows that the volumetric efficiency also increases with the increase of the tip speed. By increasing the speed the indicated power will increase coupled with the mechanical losses leading to a great increase in the input power, Fig. 7-c. As the mass flow rate is becoming larger getting the isentropic power to increase by its turn. On the other hand, the increase in the input power is larger than that of the isentropic one. This means that the total efficiency will decrease with the increase of the tip speed. This is clearly presented in Fig. 7-d.

The difference between the mass flow rate for the $5 / 7$ profile and $3 / 5$ profile is larger at the high tip speed. This is due to the great number of compression chamber formed for $5 / 7$ configuration over the $3 / 5$. As the mass flow rate increases meaning that the load have been increased also the deviation of the input power for the $5 / 7$ configuration from that of the $3 / 5$ profile is similar to that of the mass flow rate, Fig.7a. The reverse trend of the total efficiency from the profile of the input power can be remarked from Fig. 7-d.

The effects of varying the suction pressure on the compressor performance are presented in Fig.8. Figure 8-a shows that as the suction pressure increases the mass flow rate increases. Further, as a result of this relation, the volumetric efficiency also slightly increases, Fig.8-b. In addition, as both the suction pressure and the mass flow rate increase this lead to an increase in the input power in a similar manner as the mass flow rate, Fig.8-c. On the other hand, the increase in the isentropic power due to the change in the mass flow rate is greater than that of the input power which leads to a decrease in the total efficiency in the manner shown in Fig. 8-d.

The discharge pressure is the pressure with which the mixture of the working fluid with the compressed gas leaves the compressor housing. The effects of varying this pressure on the compressor performance are presented in Fig.9. It is observed from Fig.9-a that the mass flow rate remains approximately constant through the variation range. As a result, Fig.9-b shows that the volumetric efficiency also remains constant through the same range. As the delivery pressure increases, the indicated power increases causing an increase in the input power in the way shown in Fig. 9-c. Meanwhile, Fig. 9-d shows that the total efficiency is increased due to the increase of discharge pressure. The reason for this trend is the increase that happened in the delivery pressure due to increasing the condensing temperature. Consequently, the isentropic power is increased causing the increase of the total efficiency. 


\section{CONCLUSIONS}

In the present study a simulation code is used to study the effect of various geometric as well as operating parameters on the performance of oil-injected twinrotors screw compressor. The following concluding remarks can be drawn from the present study:

- As the clearance gap increases, the mass flow, the input power, and both the volumetric and total efficiencies decrease.

- Increasing the number of lobes to gates results in an increase in the mass flow rate and input power, decrease in volumetric and total efficiencies.

- Increasing the compressor relative length results in increasing the mass flow rate as well as the volumetric efficiency.

- Increasing the screw wrap angle leads to a decrease in mass flow rate.

- Increasing the tip speed leads to an increase in the mass flow rate, volumetric efficiency and input power, a decrease in total efficiency.

- The mass flow rate increases with the increase of the suction pressure.

- The variation of the discharge pressure has negligible effect on the mass flow rate.

- The compressor total efficiency increases with the decrease of suction pressure and/or the increase of the discharge pressure.

\section{ACKNOWLEDGMENTS}

The authors would like to thank Prof. Nikola Stosic for providing the SCORPATH program.

\section{REFERENCES}

[1]. Tang, Y., "Computer aided design of twin screw compressors," Ph.D. thesis, The University of Strathclyde, UK, 1995.

[2]. Chung-Biau and Shinn-Liang, "Design of pencil-type milling cutters for worm surface generation," Journal of materials processing technology, Vol. 42, pp. 361-376, 1994.

[3]. Fleming, J. S., Tang, Y., and Cook, G., "The twin helical screw compressor, Part 1: development, applications and competitive position," Proc. Instn. Mech. Engrs., Vol. 212, Part C, pp. 355-363, IMechE 1998.

[4]. Fleming, J. S., Tang, Y., and Cook, G., "The twin helical screw compressor, Part 2: a mathematical model of the working process," Roc. Instn. Mech. Engrs., Vol. 212, Part C, pp. 369-379, IMechE 1998.

[5]. Xiao, D. Z. and Li, K. B., "Computer aided design software package for conjugate helical surfaces," Journal of materials processing technology, Vol. 61, pp.72-77, 1996.

[6]. Stosic, N. and Hanjalic, K., "Development and optimization of screw machines with a simulation model - part 1: Profile generation," Journal of fluids engineering, Vol.119, pp.659-663, 1997. 
[7]. Stosic, N. and Hanjalic, K., "Development and optimization of screw machines with a simulation model - part 2: Thermodynamic performance simulation and design optimization," Journal of fluids engineering, Vol.119, pp.664-670, 1997.

[8]. Stosic, N., "On gearing of helical screw compressor rotors," Proc Instn Mech Engrs, Vol. 212, Part C, pp587-594, IMechE 1998.

[9]. Zimmern B. and Sweetser, R. S., "The centrifugal economizer its match with screw compressors," ASHRAE Trans, pp225-235, 1986.

[10]. Pillis, J. W., "Advancement in refrigeration screw compressor design," ASHRAE Trans, pp219-224,1986.

[11]. Elgohary, H. A. "Analysis of a screw-compressor performance," M.Sc. thesis, Mechanical Engineering Department, The University of Alexandria, EGYPT, 2003.

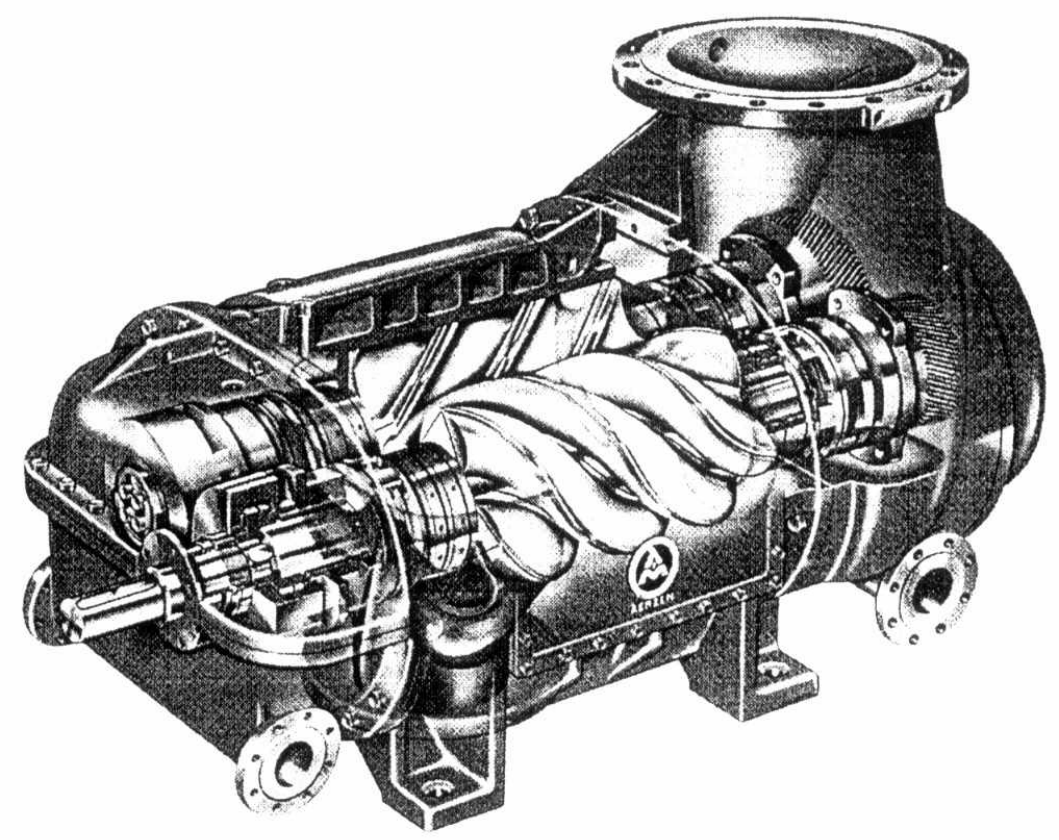

Figure 1. Rotary screw compressor (double-helical screw machine). (Aerzen USA Company, Coatesville, Pa.) 


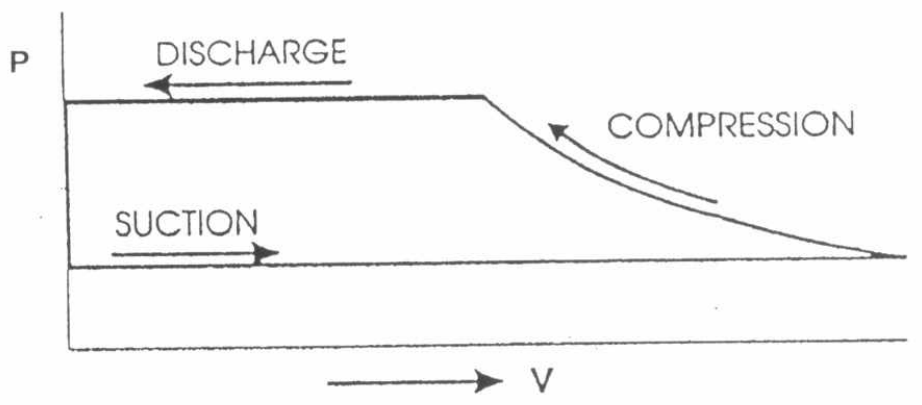

(a) Suction phase

(b) Transfer phase
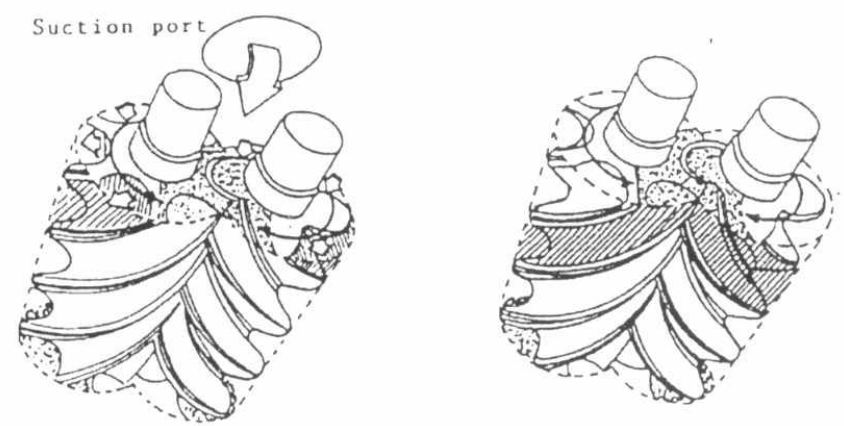

(c) Compression phase

(d) Discharge phase
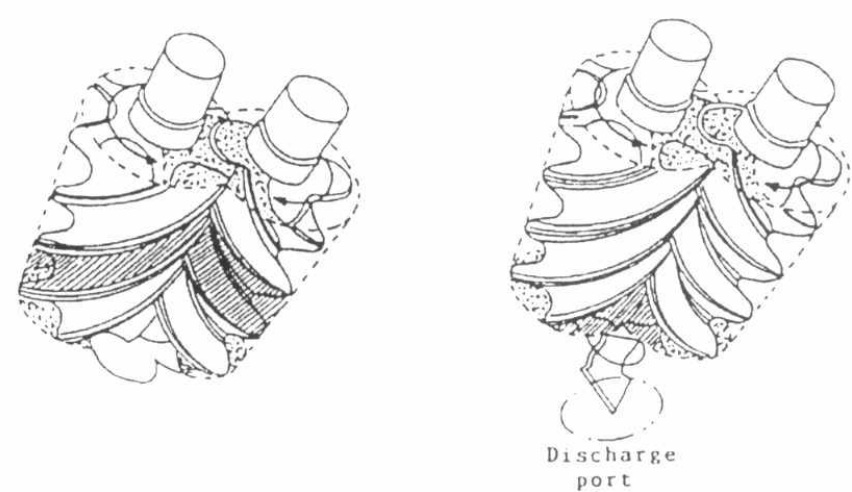

Figure 2. Working phases of rotary screw compressor. 

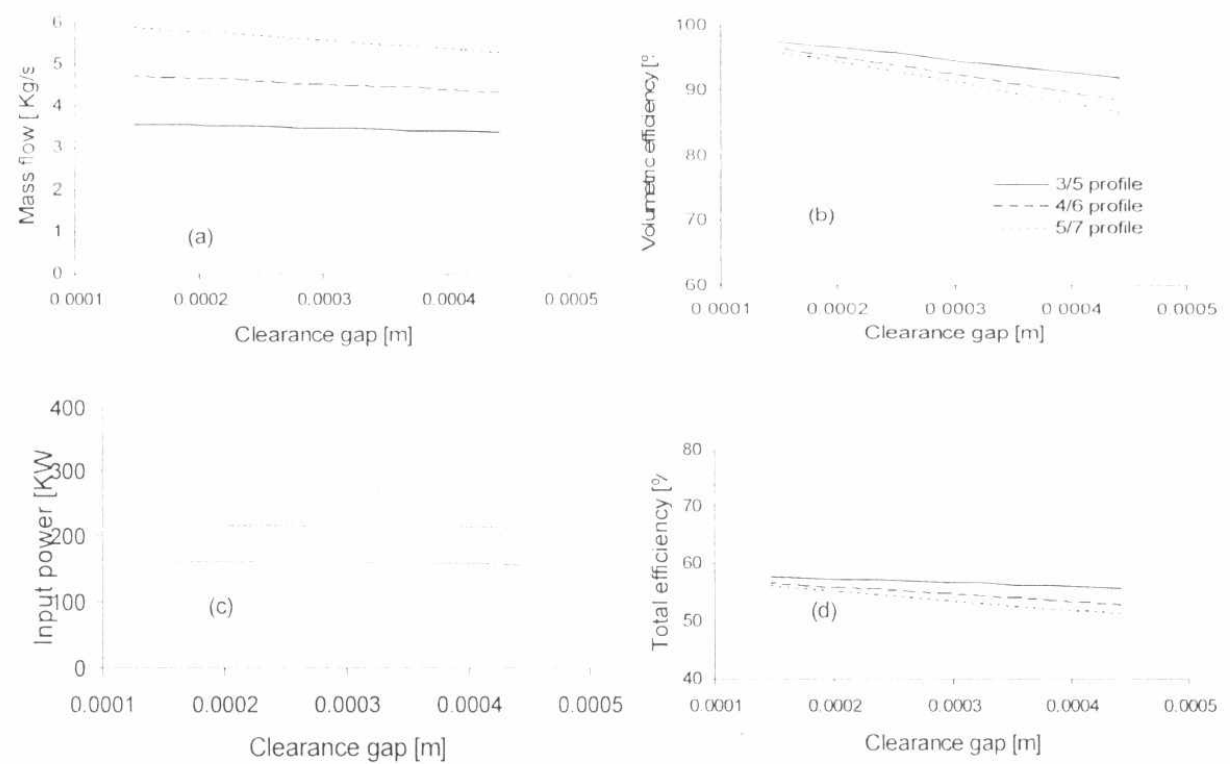

Figure 3. Variation of the clearance gap for different profile configuration with (a) Mass flow (b) Volumetric efficiency (c) Input power (d) Total efficiency
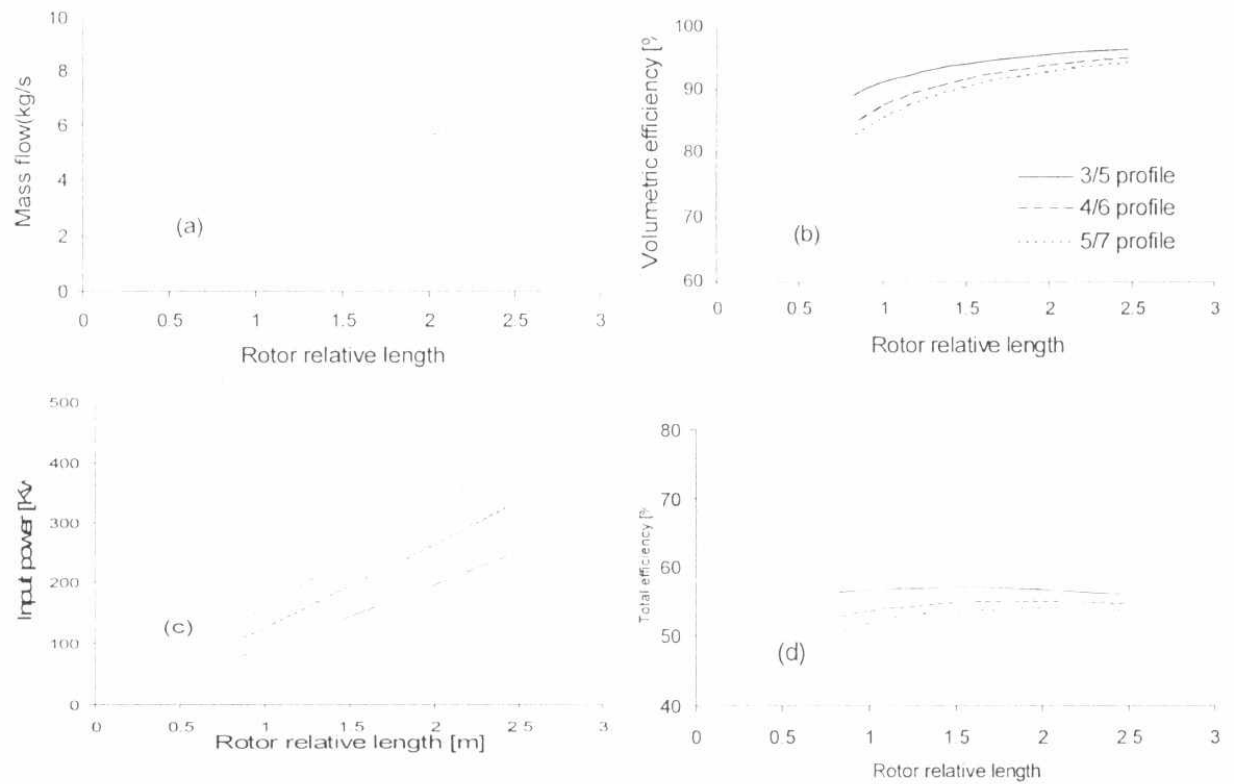

Figure 4. Variation of the rotor relative length for different profile configuration with (a) Mass flow (b) Volumetric efficiency (c) Input power (d) Total efficiency 

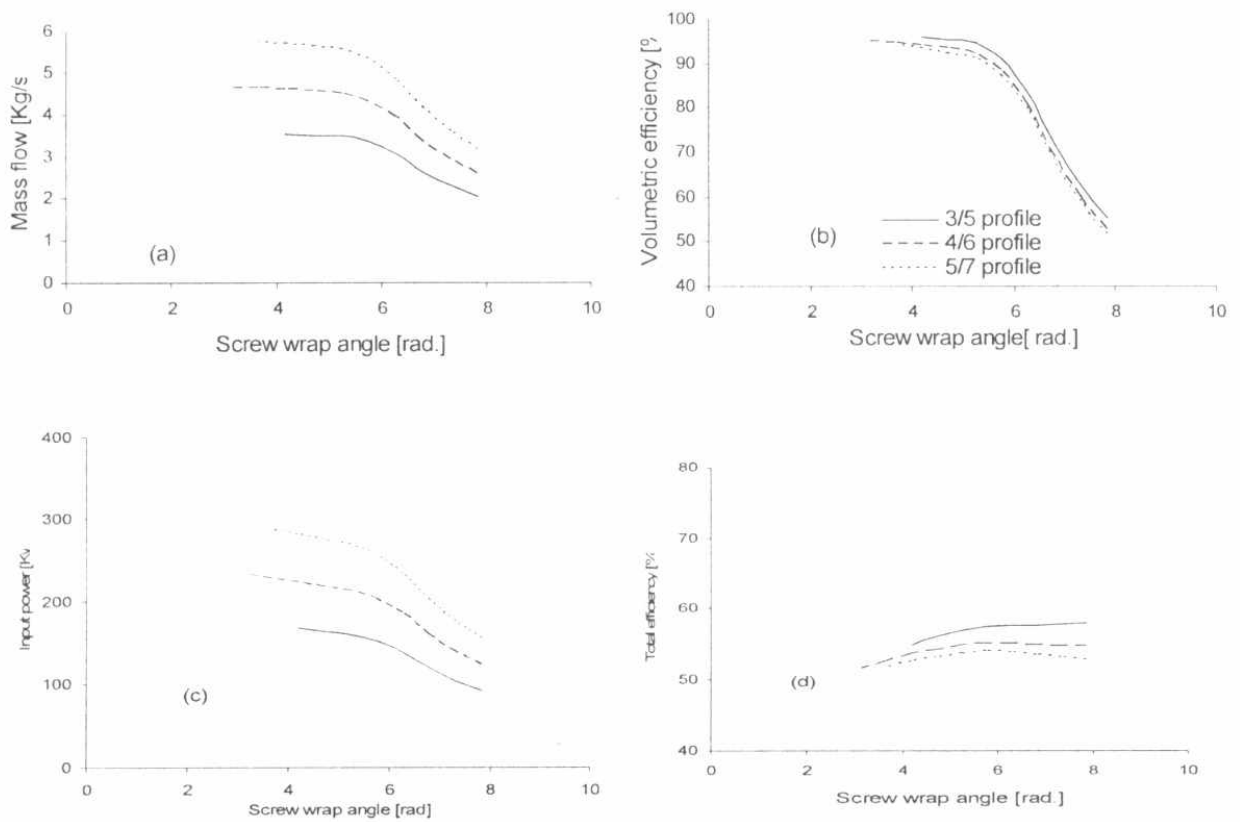

Figure 5. Variation of the screw wrap angle for different profile configuration with

(a) Mass flow (b) Volumetric efficiency (c) Input power (d) Total efficiency
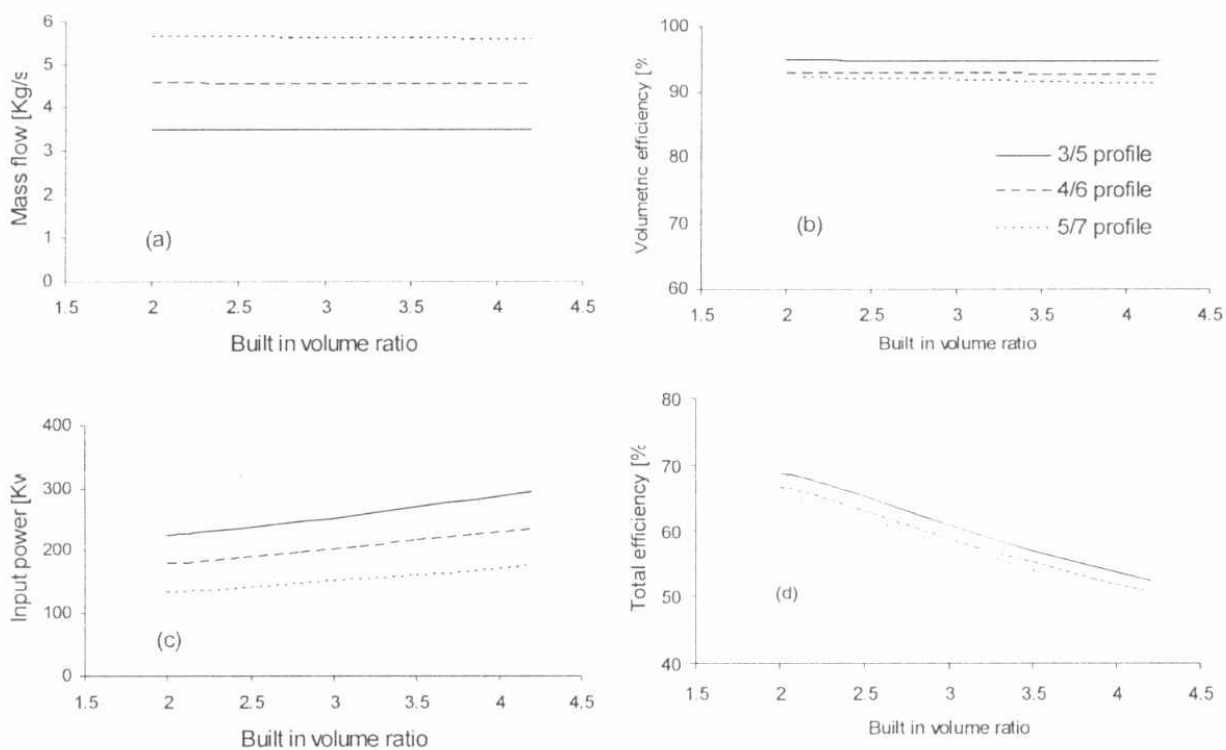

Figure 6. Variation of the built in volume ratio for different profile configuration with (a) Mass flow (b) Volumetric efficiency (c) Input power (d) Total efficiency 

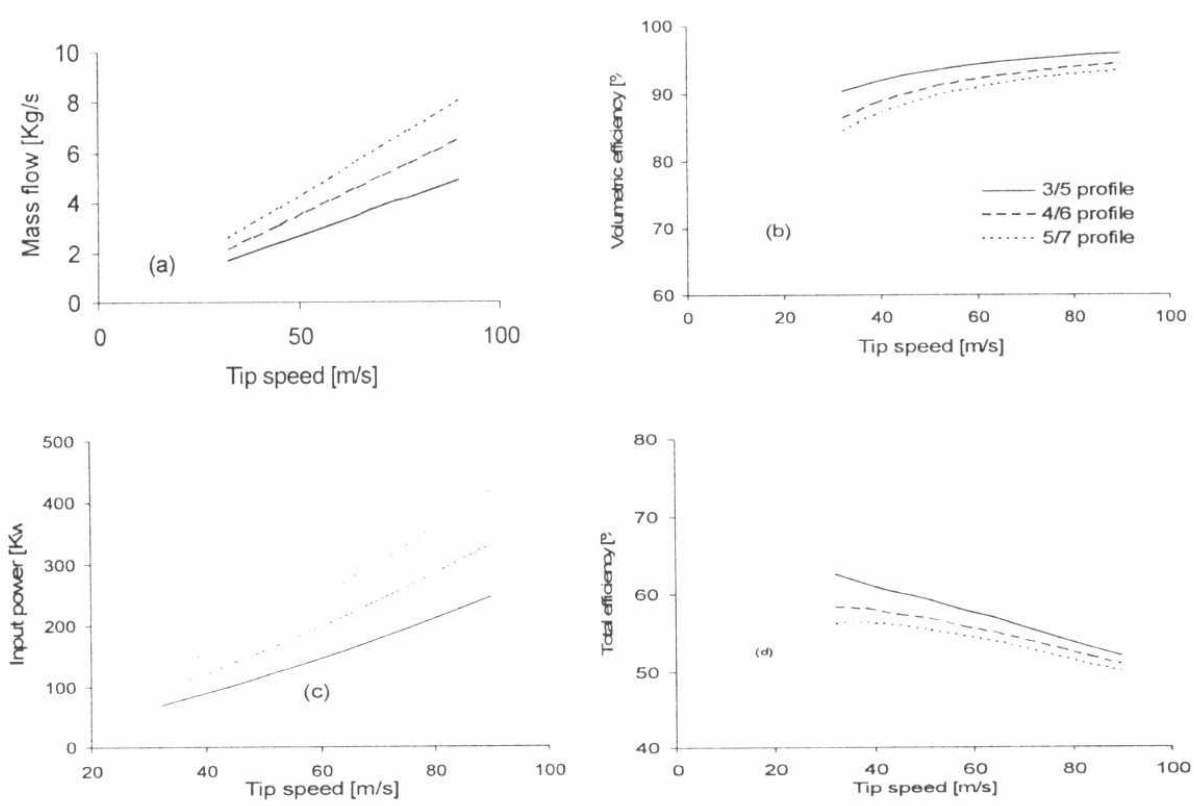

Figure 7. Variation of the tip speed for different profile configuration with (a) Mass flow (b) Volumetric efficiency (c) Input power (d) Total efficiency
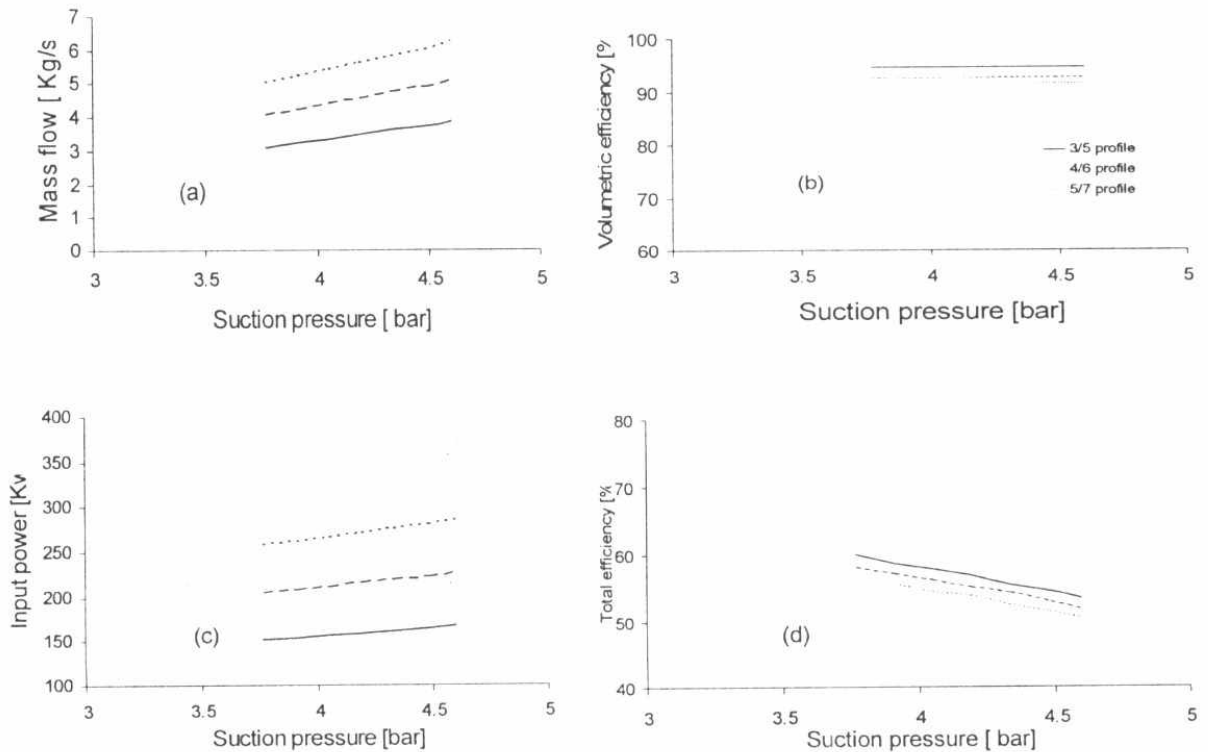

Figure 8. Variation of the suction pressure for different profile configuration with (a) Mass flow (b) Volumetric efficiency (c) Input power (d) Total efficiency 

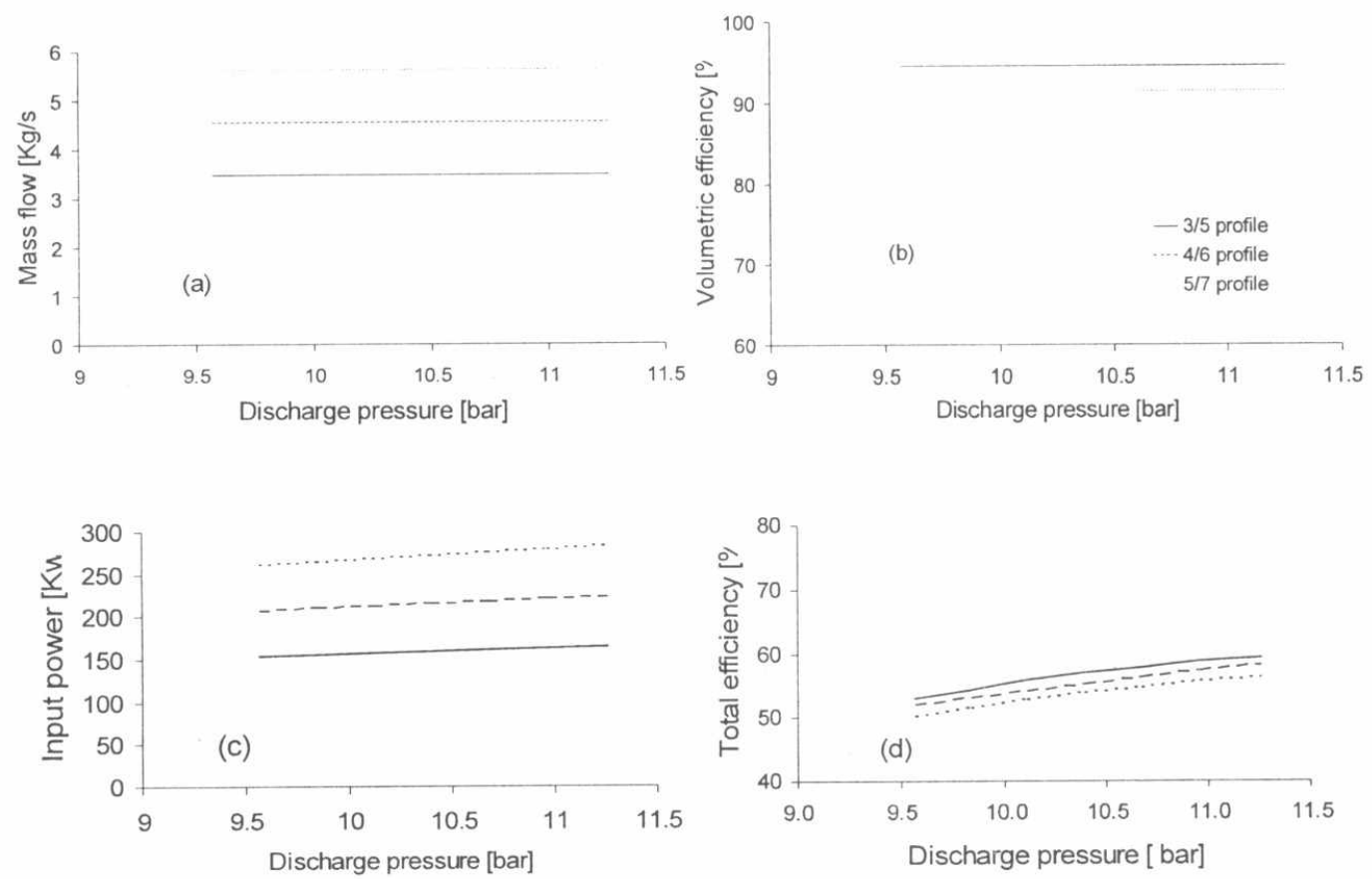

Figure 9. Variation of the discharge pressure for different profile configuration with

(a) Mass flow (b) Volumetric efficiency (c) Input power (d) Total efficiency 